\title{
STATIC CHARACTERISTICS OF AIR GAUGES APPLIED IN THE ROUNDNESS ASSESSMENT
}

\section{Czesław J. Jermak, Mirosław Rucki}

Poznan University of Technology, Institute of Mechanical Technology, Piotrowo 3, 60-965 Poznań, Poland (\czeslaw.jermak@put.poznan.pl,+48616653570,office_mt@put.poznan.pl)

\begin{abstract}
In the article, analysis of the work conditions and the metrological characteristics of the air gauges dedicated for the roundness assessment is described. To reach the required accuracy of the acquired data, the phenomena of gas dynamics had to be analyzed in the whole flow through elements of the air gauge. A model based on the second critical parameters was used, because it reflects the true processes of the air flow. As a result, fast and accurate simulations provided series of the characteristics to be considered. Nevertheless, the chosen air gauge configuration underwent the experimental verification of its metrological characteristics. Finally, the entire measurement system Geoform with the gauge head based on the chosen air gauges underwent the accuracy test in order to make sure of its overall measurement quality.
\end{abstract}

Keywords: air gauges, out-of-roundness, static characteristics, multiplication.

C 2016 Polish Academy of Sciences. All rights reserved

\section{Introduction}

"As electronics are increasingly used as the 'brains' of automatic machining, so air-operated gauges will be chosen as the 'eyes', to assess and signal or send impulses, according to how the process is running." This sentence written almost 60 years ago [1], is still valid. The air gauges were successfully applied in the first decades of the 21 st century in the industrial precise measuring tasks, like in-process inspection [2], both passive [3] and active type [4], as well as in analysis of the geometrical features like roughness [5] or form error [6]. The rapid development of computers and numerical calculation made it possible to use more precise and more sophisticated methods for data processing that were too complicated to be employed in the past [7]. That, in turn, forced science to find ways for further improvement of the metrological characteristics of air gauges [8].

The form errors of cylindrical elements are very important features, and they should be measured not only during the machining process, but also afterwards, when the detail is exploited [9]. Thus, in order to enable a non-contact, fast, relatively cheap and reasonably accurate form measurement of the cylinders, the air gauges were applied in the automatic measurement device.

\section{Basic definitions and parameters}

A simple one-cascade back-pressure gauge consists of two nozzles of diameters $d_{w}$ (the inlet nozzle marked 1 in Fig. 1, left) and $d_{p}$ (the measuring nozzle marked 3). Pressured air of pressure $p_{z}$ enters the measuring chamber (2) of a certain volume through the inlet nozzle, and leaves through the measuring one. Here, the outflow is restricted by the surface of measured detail (4) which forms a flapper-nozzle area, so the pressure $p_{k}$ in the chamber corresponds to 
the displacement $s$. The area of proportional function $p_{k}=f(s)$ is used as a measuring range $z_{p}$ (Fig. 1, right). From the measurement accuracy perspective, the declination of the proportional part of characteristics is of great importance and is called the sensitivity or multiplication $K$. The sensitivity may reveal some discontinuity which has been the subject of examinations for some 50 years [10]. The volume $V_{k}$ of the measuring chamber (2) could be reduced below $3 \mathrm{~cm}^{3}$ in order to obtain good dynamic characteristics [11].
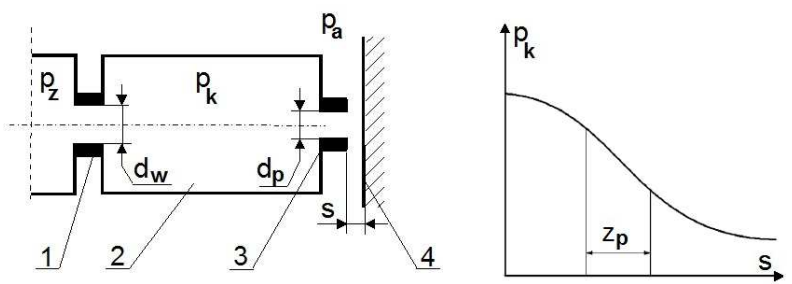

Fig. 1. An air gauge - its scheme (left) and static characteristics (right).

Mathematically, the static characteristics of air gauge is a function of its several geometrical parameters and the feeding pressure (gauge pressure), which may be written as follows:

$$
p_{k}=f\left(p_{z}, d_{w}, d_{p}, s\right)
$$

which is simplified and does not include many other parameters like the shape and length of flow-through elements, the outer diameter of measuring nozzle, roughness of the inner surfaces, etc. Those parameters have a certain impact on the flow-through coefficients of inlet nozzle and flapper-nozzle area, $\alpha_{w}$ and $\alpha_{p s}$, respectively, which are the quantitative measure of the flow process irreversibility. Thus, the stream continuity means that the mass flow through the inlet nozzle $\dot{m}_{w}$ should be equal to the mass flow through the flapper-nozzle area $\dot{m}_{p s}$. The stream continuity equation could be written:

$$
\dot{m}_{w}=f_{1}\left(d_{w}, \alpha_{w}, p_{k}, p_{z}\right)=\dot{m}_{p s}=f_{2}\left(d_{p}, \alpha_{p s}, s, p_{k}, p_{a}\right) .
$$

In the case of inlet nozzles, it could be either the regulated flow surface or the set diameter. In the last case, the static characteristics is more stable, and the metrological parameters of air gauge (sensitivity and measuring range) could be changed by replacement of the inlet nozzle. The most common diameters of inlet nozzles are between 0.5 and $2.0 \mathrm{~mm}$. Smaller nozzles are more economical because they provide a smaller mass flow. However, the dynamic characteristics of air gauge worsens [11].

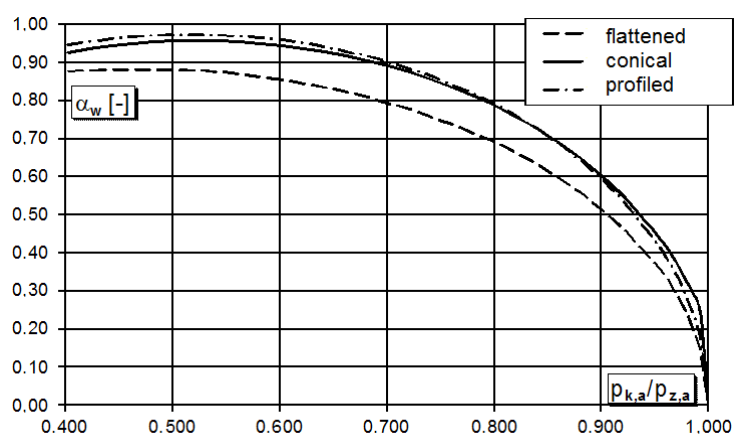

Fig. 2. The flow coefficient as a function of normalized values of the absolute back-pressure for different nozzles. 
The inlet nozzle surface can be left flat, or conical, or profiled, which influences the flow coefficient $\alpha_{w}$. As it is seen in the diagram (Fig. 2), the difference between profiled and conical nozzles is insignificant, while the profiled one is much more expensive to be shaped.

\section{Flow through air gauge elements}

It became clear as early as in the middle of the 20th century that the simplicity in functioning and using air-operated gauges in no way implied that the technical considerations involved in the choice resulted in obtaining the required flow characteristics [12]. Numerous matematical models have been applied to calculate the static characteristics of air gauges [13]. Each of them is based on the formulas of flow through the inlet nozzle and in the flapper-nozzle area [10]. The differences between the models lay basically in the loss coefficients and in the way of presenting the function itself. Some authors propose to assume incompressibility of air, and even though they provide relatively good results, especially for the small pressure falls, the true nature of the flow is not reflected [11].

Apart of the turbulent flow, in the air gauges could appear a supersonic flow, as well as strike waves which absorb the flow energy. Hence, the true mass flow is different from the theoretical one which can be calculated with the Saint Venant - Wantzel discharge formula [10]:

$$
\dot{m}_{t}=A \frac{P_{0}}{\sqrt{R T_{0}}} \sqrt{\frac{2 \kappa}{\kappa-1}} \sqrt{\left(\frac{P}{P_{0}}\right)^{\frac{2}{\kappa}}-\left(\frac{P}{P_{0}}\right)^{\frac{\kappa+1}{\kappa}}},
$$

where: $\dot{m}_{t}$ - the theoretical mass flow; $A$ - the outflow surface; $P_{0}, T_{0}$ - the absolute pressure and temperature in the stagnation point at the nozzle inlet; $P$ - the absolute pressure in the nozzle outlet intersection; $\kappa$ - the isentropic factor; $R$ - the gas constant (in the air gauges it is usually air).

The pressure ratio which corresponds to the maximal mass flow $\dot{m}_{t}=\dot{m}_{t \max }$ is called the critical ratio:

$$
\beta_{k r 1}=\frac{P_{k r 1}}{P_{0}}=\left(\frac{2}{\kappa+1}\right)^{\frac{\kappa}{\kappa-1}},
$$

where $P_{k r 1}$ is the first critical pressure (absolute), when the outlet mass flow is maximal.

Because the stream contraction causes some loss of energy, the actual pressure ratio for maximal mass flow differs from the theoretical one. It is called the second critical ratio $\beta_{k r}$. The corresponding flow coefficient is:

$$
\alpha_{k r 2}=\frac{\dot{m}_{r z \max }}{\dot{m}_{t \max }},
$$

where $\dot{m}_{r z \max }, \dot{m}_{t \max }$ represent the actual and theoretical maximal mass flows, respectively.

Figure 3 presents the normalized mass flow graphs as functions of the absolute pressure ratio $\beta=P / P_{0}$. The curve 1 corresponds to the ideal isentropic flow, where the maximal mass flow is reached for the pressure ratio $\beta=0.528$; the other curves represent the real mass flows for different nozzles: profiled (2), conical (3) and flattened (4). The simplified formula for calculations of the flow coefficient based on the second critical parameters is following [10]:

$$
\alpha=\frac{1}{m_{t}} \frac{\alpha_{k r 2}}{1-\beta_{k r 2}} \sqrt{1-2 \beta_{k r 2}(1-\beta)-\beta^{2}} .
$$


The formula (6) could be written in a more common form by applying the Sanville flow function $\Psi(\beta)$, which is widely used for mathematical modelling of the air mass flow through the local pneumatic resistance. The flow function may be described as follows [14]:

$$
\Psi(\beta)=\left\{\begin{array}{ll}
1 & \text { for } \beta \leq \beta_{k 2} \\
\sqrt{1-\left(\frac{\beta-\beta_{k 2}}{1-\beta_{k 2}}\right)^{2}} & \text { for } \beta_{k 2}<\beta \leq 1
\end{array}\right. \text {. }
$$

The results obtained from (6) revealed good conformity with the experimental data, and could be applied both to the inlet and measuring nozzles. In the last case, however, the energy loss in the nozzle orifice contains ca. $4 \%$ of the entire loss in the flapper-nozzle area [10].

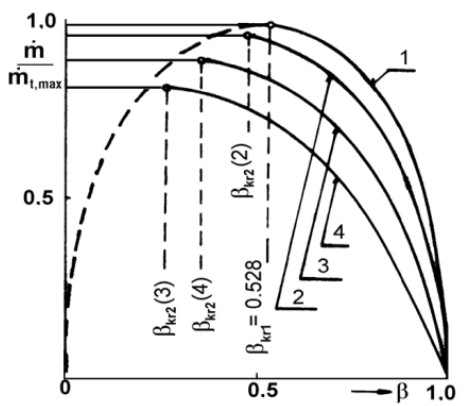

Fig. 3. The theoretical and actual mass flows as functions of the pressure ratio $\beta$.

Figure 4 presents the areas where the losses appear: in the nozzle orifice described by the coefficients $\zeta_{1}, \zeta_{2}$ and $\zeta_{3}$, and in the area between the nozzle head and the flapper surface described by the coefficients $\zeta_{4}, \zeta_{5}$ and $\zeta_{6}$. To express the overall pressure loss $\zeta_{\mathrm{c}}$ related to the mean pressure in the measuring slot $s$ (limited by the outer diameter $d_{c}$ ) the following formula was proposed [10]:

$$
\zeta_{c}=16 \frac{d_{c}^{2} \cdot s^{2}}{d_{p}^{4}}\left(\zeta_{1}+\zeta_{2}+\zeta_{3}\right)+\left(\left[\frac{d_{c}}{d_{p}}\right]^{2} \zeta_{4}+\zeta_{5}+\zeta_{6}\right)
$$

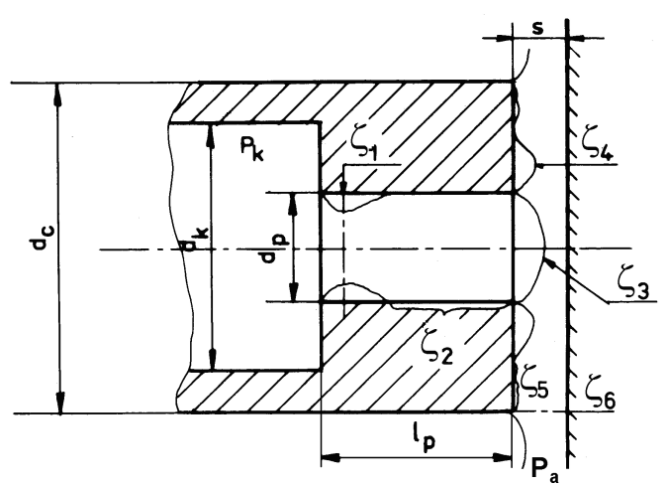

Fig. 4. The pressure losses in the flapper-nozzle area [10]. 
As it has been proved, the area limited by the outer diameter $d_{c}$ and the flapper surface may generate some phenomena that have a negative impact on the metrological properties of air gauges [15]. In particular, a wide nozzle head may generate discontinuity of the static characteristics which could substantially shorten the measuring range.

The area between the inlet and measuring nozzles is a measuring chamber, where the backpressure is measured, assuming knowledge of the slot width $s$, and, hence, of the measured dimension. The role of a measuring chamber could play either a long hose connecting the gauge head with the main device, or a reduced pneumatic cascade placed entirely in the gauge head [16]. The proposed mathematical model for a non-stationary flow through the air gauge measuring chamber, based on the Reynolds equations and the $k-\varepsilon$ turbulence model [17], provided the information on the flow inside the measuring chamber. Figs. 5 and 6 present the velocity profiles and the velocity vectors inside the air gauge measuring chamber, respectively.

The turbulences and backward directed flow are the sources of the pressure fluctuation which results in an unstable measuring signal registered in Fig. 7 for the middle of the measuring range of air gauge of $d_{p}=1.610 \mathrm{~mm}, d_{w}=1.210 \mathrm{~mm}, D_{C}=d_{c} / d_{p}=1.5$. The number of registered subsequent measurements was $N=29,566$.

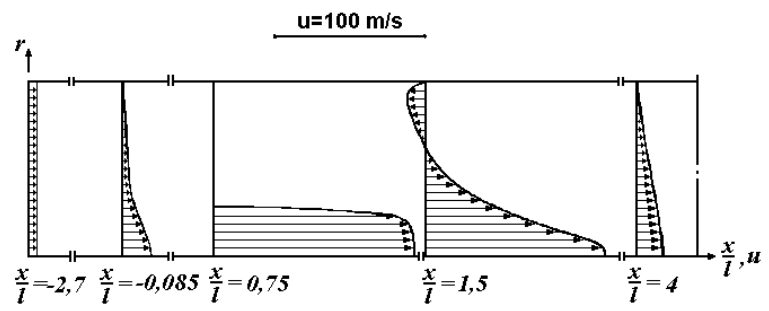

Fig. 5. The velocity profiles inside the air gauge measuring chamber [17].

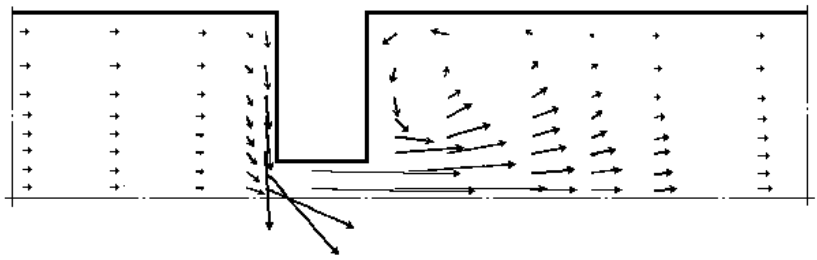

Fig. 6. The velocity vectors inside the air gauge measuring chamber [17]

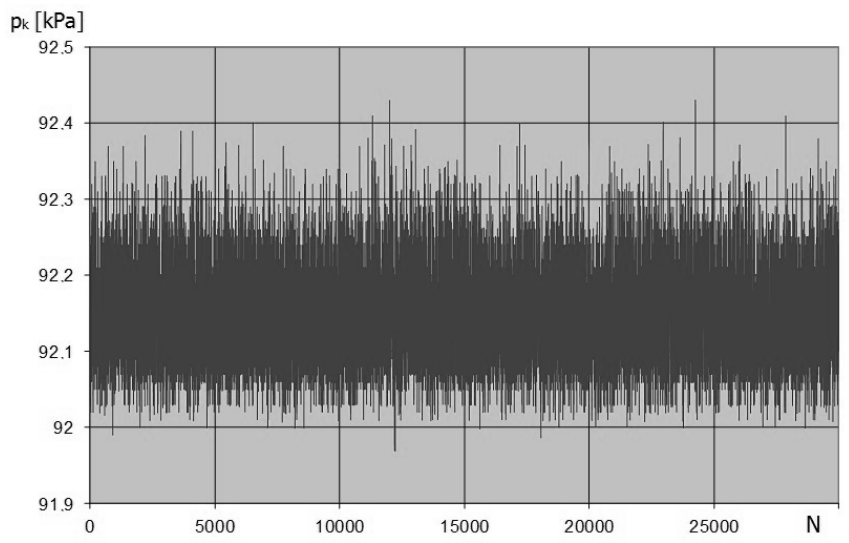

Fig. 7. The registered pressure fluctuations in the measuring chamber. 
Based on the above knowledge, it is possible to perform simulations of the air gague static characteristics [18], as well as the non-stationary flows [17].

\section{Simulation and experimental results}

In order to choose proper configurations of air gauges for a particular task of the roundness measurement, a series of simulations were performed. The following example illustrates the main tendencies in the static characteristics of the air gauges. Figs. 8 and 9 show the simulated static characteristics (left) and their multiplications (right) for a set with $d_{p}=2.003 \mathrm{~mm}$ combined with $d_{w}=0.830 \mathrm{~mm}, 1.020 \mathrm{~mm}$ and $1.210 \mathrm{~mm}$. The assumed feeding pressure was $p_{z}=150 \mathrm{kPa}$.
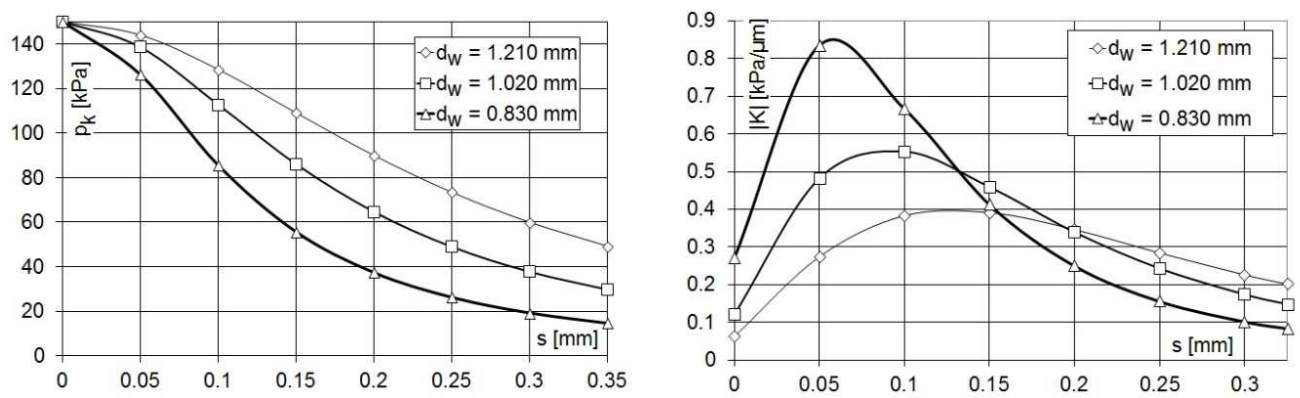

Fig. 8. The simulation results of static characteristics (left) and multiplication (right).
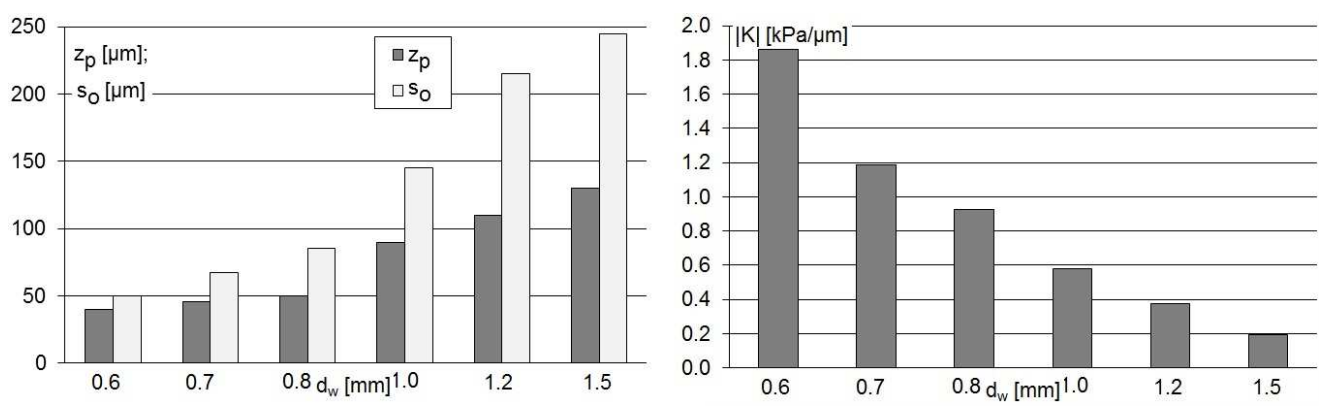

Fig. 9. The simulation results of measuring range $z_{p}$ and its middle point $s_{0}$ (left) and multiplication (right).

After the initial choice of the projected air gauges, their characteristics underwent experimental verification and correction. The laboratory equipment for analysis of static characteristics has been described in detail in [10], and its scheme is shown in Fig. 10.

It consists of:

1. An initial air pressure reducer LPR type made by FESTO, equipped with a filter and a valve.

2. A precise pressure stabilizer, EIR type (made by SMC company), equipped with an additional device for pressure calibration 717 30G (FLUKE).

3. An electronic manometer SMRF-EB made by Sensyn company.

4. The examined air gauge.

5. A piezo-resistive pressure transducer 4043A5 type, produced by Kistler AG, combined with a Kistler amplifier 4601A.

6. A measuring column TT 500 connected with an inductive sensor GT21HP. 
7. A digital voltmeter V530 made by Mera Tronic.

8. A moving table for fastening the parts of equipment.

9. A flapper surface (measured object).
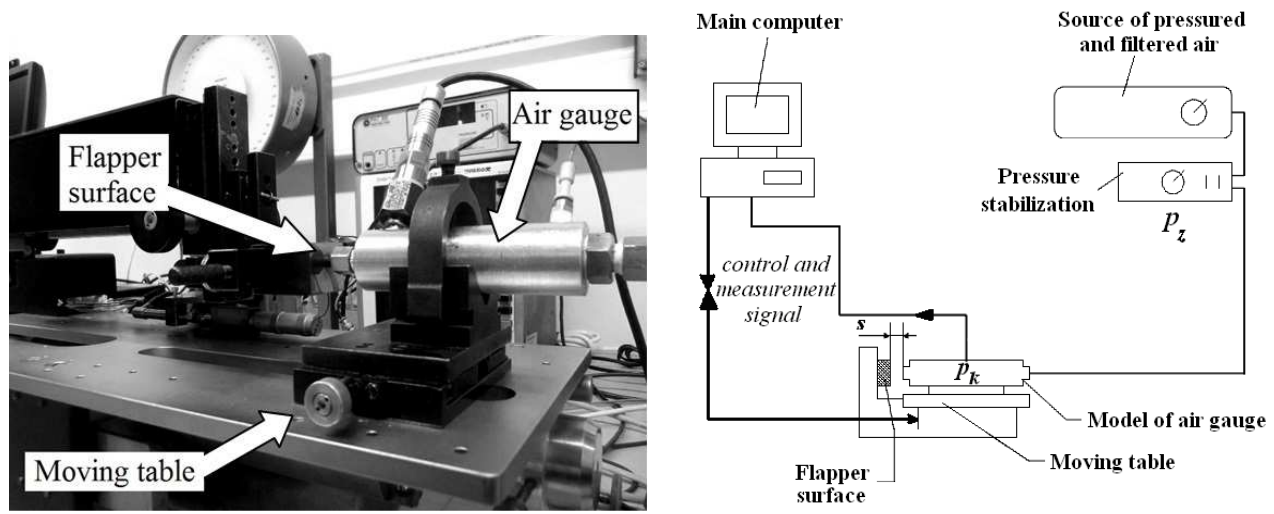

Fig. 10. A scheme of laboratory equipment for measurement of the air gauge static characteristics [19].

The whole set is controlled by a PC equipped with StanBad program. The initial and final slot is input, the sampling step may be chosen, the number of repetitions may be given. After the data are collected, they are processed with another program - CharStat, which presents the results of measurement both numerically and graphically.

Because the measurement task was to assess out-of-roundness of the cylinder with the tolerance of $10 \div 15 \mu \mathrm{m}$, the following geometrical parameters of the air gauge were chosen: the measuring nozzle with the orifice diameter $d_{p}=1.610 \mathrm{~mm}$ and the outer diameter $d_{c}=4.8$, the inlet nozzle with the diameter $d_{w}=1.020 \mathrm{~mm}$. Geometry of those nozzles is presented in detail in Fig. 11 . The conical entrance of measuring nozzle was designed to smoothen the phenomena of gas dynamics discussed above. This configuration was able to perform the measurement with multiplication $|K|=0.5 \mathrm{kPa} / \mu \mathrm{m}$ within the range of $z_{p}=100 \mu \mathrm{m}$ with non-linearity not exceeding $0.5 \%$. Fig. 12 presents the experimentally obtained characteristics of a chosen air gauge.
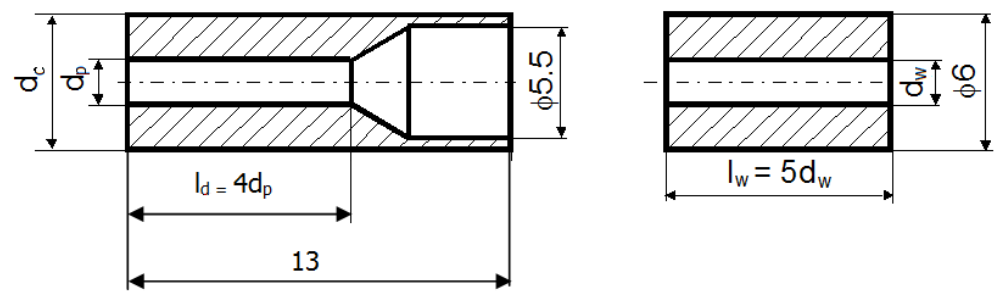

Fig. 11. The dimensions of chosen nozzles: measuring (left) and inlet (right). 


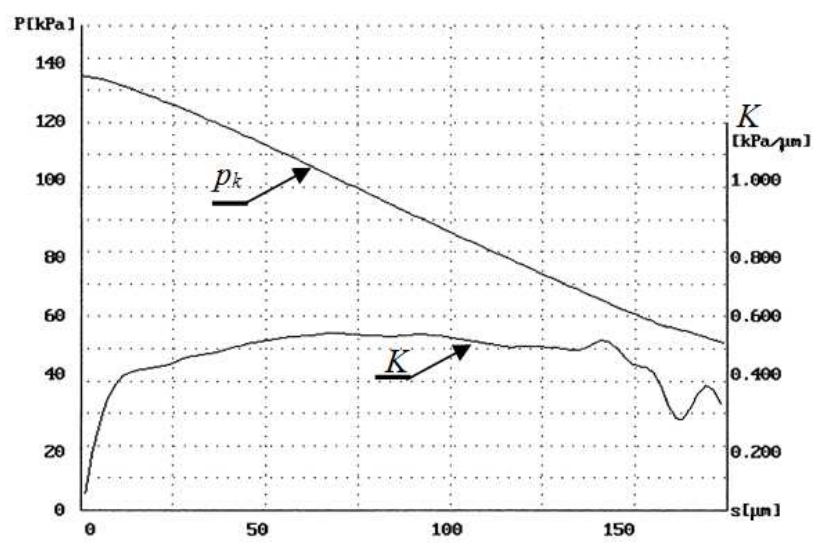

Fig. 12. The experimental static characteristics of a chosen air gauge.

\section{The measurement of roundness}

The chosen air gauges were built into a patented gauging head (patent PL 211360) of the GEOFORM device designed to measure the diameter and roundness of inner surfaces like rings, sleeves or cylindrical openings (Fig. 13). The operator decides on how many levels the measurement should be performed. Before the measurement, the device should be calibrated with a setting ring. The results of measurement are presented as a set of numbers, and as a circle graph. The software enables also to perform harmonic analysis of amplitudes in the measured intersection.

The device is capable to measure inner diameters of $d>100 \mathrm{~mm}$ with the uncertainty of $U_{k=2}< \pm 1.5 \mu \mathrm{m}$. The measurement results are presented on the screen both numerically and graphically. In the example presented in Fig. 14, the measurement results of roundness at level 2 (in the middle of the cylinder) are presented in the scale from -10 to $+10 \mu \mathrm{m}$. The obtained out-of-roundness is $8.1 \mu \mathrm{m}$.
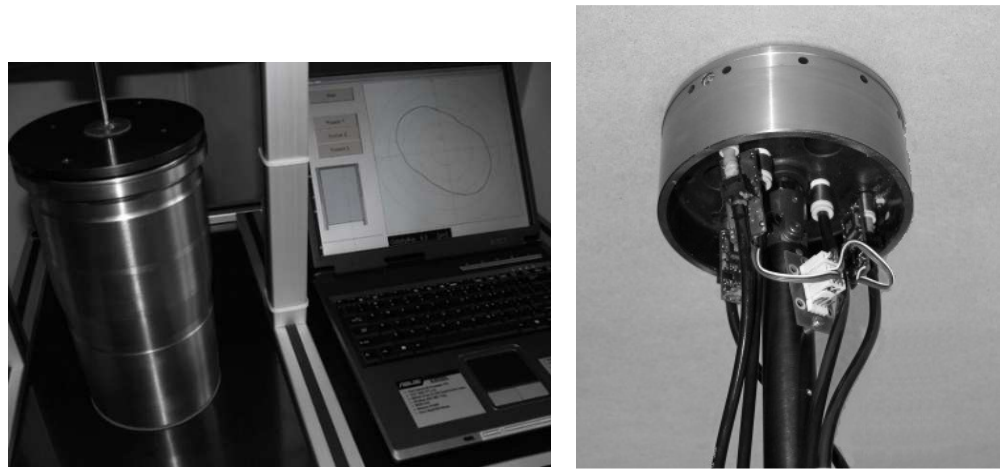

Fig. 13. A view of the Geoform device (left) and the gauging head (right) 


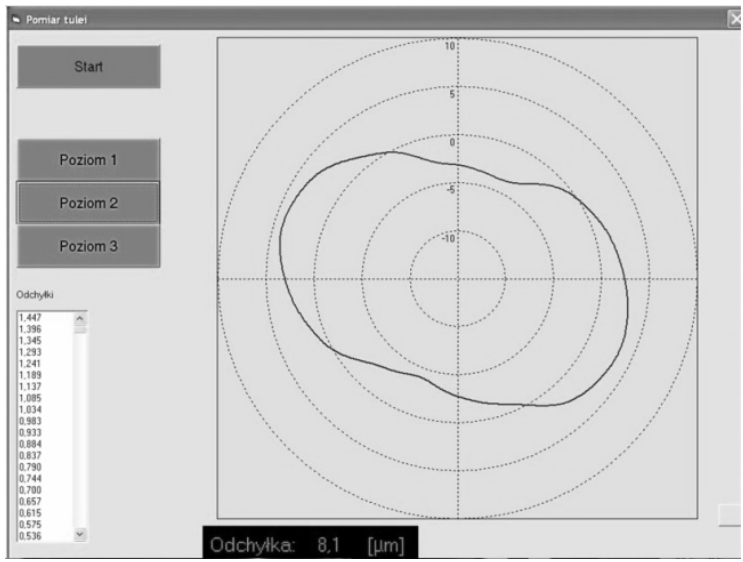

Fig. 14. An example of the measurement results presented on the screen [10].

The applied idea of assessing the roundness is very different from a typical three-point measurement described e.g. in [20]. Calculation of the profile and its out-of-roundness is performed as if a typical V-block measurement is performed, only instead of two fixed points of the V-block, the data collected from two air gauges are used. Thus, instead of one measurement result $\Delta W$, the gauge head provides three results: $\Delta R_{1}, \Delta R_{2}$ and $\Delta R_{3}$ from three independent air gauges, as shown in Fig. 15. Based on the principle presented in [21], the measured parameter $\Delta W$ could be calculated as:

$$
\Delta W=\Delta R_{3}+\frac{\Delta R_{1}+\Delta R_{2}}{2 \cos \alpha}
$$

where: $\Delta R_{1}, \Delta R_{2}$ and $\Delta R_{3}$ - the indications from three gauges; $2 \alpha$ - the angle between the air gauges 1 and 2 .
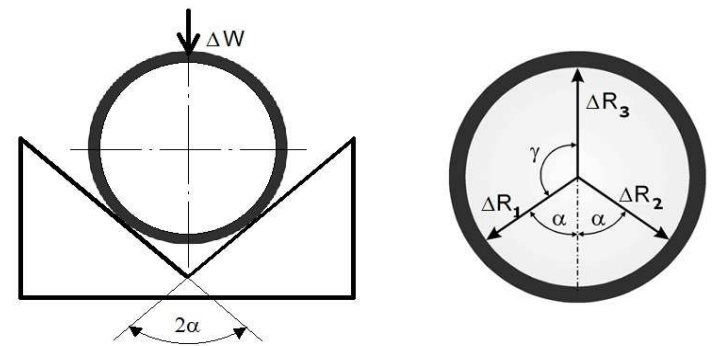

Fig. 15. The obtained results: $\Delta R_{1}, \Delta R_{2}$ and $\Delta R_{3}$, recalculated into the V-block measurement $\Delta W$.

The achieved result $\Delta W$ is then transformed into a Fourier series, and particular harmonics undergo a correction procedure. Calculation of the Fourier coefficients was based on substituting a trapezoid function in the Euler's formulas [22]. The final formulas for the harmonic analysis were following:

$$
\begin{aligned}
& n a_{m}=\sum_{k=0}^{2 n-1} \Delta W_{k} \cos \frac{k m \pi}{n} \\
& n b_{m}=\sum_{k=0}^{2 n-1} \Delta W_{k} \sin \frac{k m \pi}{n}
\end{aligned}
$$


where: $m=2,3, \ldots, 15 ; k=0,1,2, \ldots, 2 n$ (for $360^{\circ} n=360$ ).

Figure 16 illustrates that the profile identified by the Geoform device is - in general - the same as the one identified by the Talyrond 365 . All the main profile features are represented in both their quality and quantity.
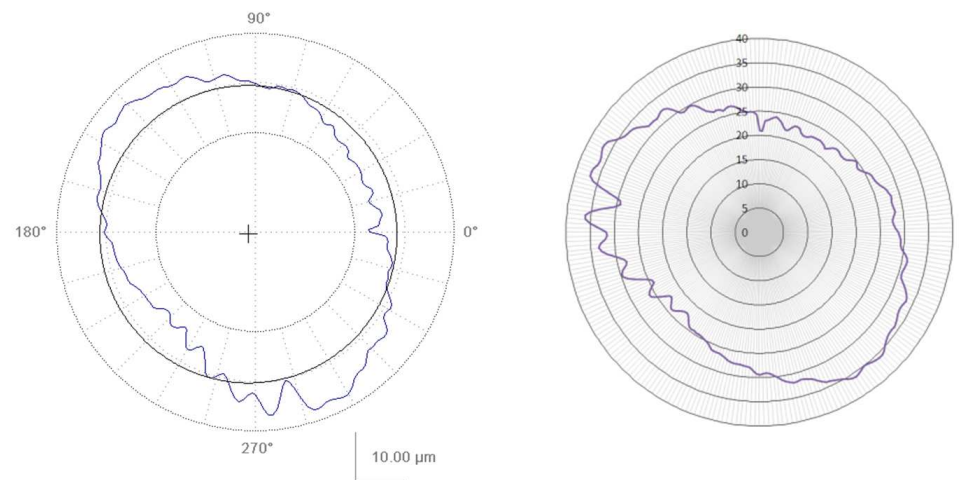

Fig. 16. The graphical representations of the same profile measured by the Talyrond 365 (left) and Geoform (right) devices.

The overall accuracy of the measurement system which determines its capability to perform the presumed measuring task was calculated according to the recommendations proposed by Adamczak [23]. For that purpose, the reference measurement data were obtained from the Talyrond 365 device. After 100 repetitions with different cylinders, the DP factor (described in detail in [24]) covering the differences between the reference measurement and the tested method was calculated to be $9.40 \%$, which is highly satisfactory.

\section{Conclusions}

The measurement of such a feature like out-of-roundness requires a high accuracy and repeatability. In the examined task, the tolerances of $10 \div 15 \mu \mathrm{m}$ were set by the industrial requirements. In order to perform a non-contact, fast measurement with a reasonable accuracy at the lowest possible price, the patented gauging head with three independent air gauges was analyzed.

To reach the required accuracy of the acquired data, the phenomena of gas dynamics had to be analyzed in the whole flow through elements of the air gauge. It could be concluded that a proper shape of the nozzles and measuring chamber may smoothen turbulences and strike waves and thus improve the metrological properties of the air gauges.

Another conclusion states that the second critical parameters provide very accurate results of the static characteristics of air gauges, because they reflect the true flow through processes. As a result, fast and accurate simulations based on a wide range of various dimensions and other parameters of the proposed air gauge provide a series of characteristics to be considered. Nevertheless, the chosen air gauge configuration underwent experimental verification of its metrological characteristics and was found suitable for the designed measuring task.

The measurement Geoform system with the gauge head based on chosen air gauges was again tested in order to make sure of its overall measurement quality and capability. The obtained result of $9.40 \%$ accuracy compared to that of the reference Talyrond 365 device proved that the air gauges built into the Geoform system perform their task with the required accuracy. 


\section{Acknowledgement}

This work was supported by the Ministry of Science and Higher Education, Poland, project N505 010 32/1555.

\section{References}

[1] Tanner, C.J. (1958). Air gauging - history and future developments. Institution of Production Engineers Journal, 37(7), 448-462.

[2] Vacharanukul, K., Mekid, S. (2005). In-Process Dimensional Inspection Sensors. Measurement, 38, 204-218.

[3] Wang, Y.H., Yu, X.F., Fei, Y.T. (2005). An Automatic Sorting System Based on Pneumatic Measurement. Key Engineering Materials, 295-296, 563-568.

[4] Menzies, I., Koshy, P. (2009). In-process detection of surface porosity in machined castings. International Journal of Machine Tools \& Manufacture, 49, 530-535.

[5] Koshy, P., Grandy, D., Klocke, F. (2011). Pneumatic non-contact topography characterization of finish-ground surfaces using multivariate projection methods. Precision Engineering, 35(2), 282-288.

[6] Liu, J., et al. (2012). Design and accuracy analysis of pneumatic gauging for form error of spool valve inner hole. Flow Measurement and Instrumentation, 23, 26-32.

[7] Janiczek, T., Janiczek, J. (2010). Linear dynamic system identification in the frequency domain using fractional derivatives. Metrol. Meas. Syst., 17(2), 279-288.

[8] Jermak, Cz.J. (2010). Methods of Shaping the Metrological Characteristics of Air Gauges. Journal of Mechanical Engineering, 6(56), 385-390.

[9] Stępien, K., Janecki, D., Adamczak, S. (2011). Investigating the influence of selected factors on results of Vblock cylindricity measurements. Measurement, 44, 767-777.

[10] Jermak, Cz.J., Rucki, M. (2012). Air Gauging: Static and Dynamic Characteristics. Barcelona: IFSA.

[11] Rucki, M., Jermak, Cz.J. (2012). Dynamic Properties of Small Chamber Air Gages. Journal of Dynamic Systems, Measurement, and Control, 134(1), 011001.

[12] Tanner, C.J. (1958). Air gauging - history and future developments. Institution of Production Engineers Journal, 37(7), 448-462.

[13] Bokov, V.B. (2011). Pneumatic gauge steady-state modelling by theoretical and empirical methods. Measurement, 44, 303-311.

[14] Kamiński, Z., Kulikowski, K. (2015). Determination of the functional and service characteristics of the pneumatic system of an agricultural tractor with mechanical brakes using simulation methods. Maintenance and Reliability, 17(3), 355-364.

[15] Jermak, Cz.J., Barisic, B., Rucki, M. (2010). Correction of the metrological properties of the pneumatic length measuring gauges through changes of the measuring nozzle head surface shape. Measurement, 43(9), 1217 1227.

[16] Rucki, M., Barisic, B., Varga, G. (2010). Air Gauges as a Part of the Dimensional Inspection Systems. Measurement, 43(1), 83-91.

[17] Jermak, Cz.J., Spyra, A., Rucki, M. (2012). Mathematical Model of Dynamic Work Conditions in the Measuring Chamber of an Air Gauge. Metrol. Meas. Syst., 19(2), 29-38.

[18] Piątkowski, R., Jermak, Cz.J., Rucki, M. (2011). The exact calculation of the air gauge static characteristics. Theory and Practice of Air Gauging. Jermak, Cz.J. (ed), Poznan University of Technology, Poznań, 43-56.

[19] Rucki, M. (2007). Step Response of the Air Gauge. Metrol. Meas. Syst., 14(3), 429-436.

[20] Gao. W., Kiyono, S. (1997). On-machine roundness measurement of cylindrical workpieces by the combined three-point method. Measurement, 21(4), 147-56. 
[21] Cellary, A., Jermak, Cz.J., Majchrowski, R. (2009). Simulation Methods for Determination of the Errors of Reference Roundness Measurement System. Proc. of the 4th International Conference Metrology in Production Engineering, Poznań, Poland, 53-58.

[22] Rudin, W. (1976). Principles of mathematical analysis. New York: McGraw-Hill, Inc.

[23] Janusiewicz, A., Adamczak, S., Makieła, W., Stepien, K. (2011). Determining the theoretical method error during an on-machine roundness measurement. Measurement, 44(9), 1761-1767.

[24] Adamczak, S., Janusiewicz, A., Makieła, W., Stępień, K. (2011). Statistical validation of the method for measuring radius variations of components on the machine tool. Metrol. Meas. Syst., 18(1), 35-46. 